\title{
Geld regiert die Politik?* Wahlkampffinanzierung in den USA
}

Kongresswahlkämpfe als privat finanzierte Unternehmungen, nur in der Theorie strikte Beschränkungen für die bloß zum Teil aus öffentlichen (Steuer-)Geldern bezahlten Präsidentschaftskampagnen mit vielen Umgehungsmöglichkeiten, extrem kostenintensive Fernsehwettbewerbe sowie ein Wahlverhalten nach sozioökonomischen Auslesekriterien - das alles führt regelmäßig zu Debatten, ob in den USA jenseits aller demokratiepolitischen (Gleichheits-)Kriterien Geld ein Schlüsselfaktor ist, der in Wahlen über Erfolg oder Misserfolg entscheidet. Die demokratische Wettbewerbspraxis wird von Kritikern seit mehr als einem Jahrzehnt als , checkbook democracy" (West 2000) bezeichnet, in welcher zahlungskräftige Geldgeber den politischen Prozess quasi gekidnappt hätten.

Spendensammeln und Kontakte mit potenziellen Geldgebern sind demzufolge bereits vom Zeitaufwand her für Kongress- und Präsidentschaftskandidaten wichtiger als das Bemühen um inhaltliche Programme. ${ }^{1}$ Entsprechende Abhängigkeiten können nicht ausgeschlossen werden. Das Hauptproblem ist jedoch die mangelnde Transparenz, weil auch unabhängig von den variantenreichen Umgehungen das Regulierungssystem der im Wahlkampf eingesetzten finanziellen Mittel in den USA sehr komplex und oft in sich widersprüchlich ist.

Bereits die Überblicksliteratur zur Wahlkampffinanzierung (campaign finance) in den USA ist extrem umfangreich und durch eine Fülle von Details gekennzeichnet. $^{2}$ Historische Entwicklungen und verfassungsrechtliche Rahmenbedingungen, detaillierte Gesetze auf allen Staatsebenen und höchstgerichtliche Entscheidungen des U.S. Supreme Court sowie die Vielfalt der Akteure (Kandidaten, Parteien, Political Action Committees/PACs usw.) bedingen vielfältige Zusammenhänge, die für die Öffentlichkeit weder transparent noch nachvollziehbar sind.

* Der Titel ist als Anlehnung entnommen aus einem Beitrag in Spiegel Online vom 5. März 2012 (http://www.spiegel.de/politik/ausland/us-wahlkampf-geld-regiert-die-politik-a-817926.html; Stand: 30.6.2012).

1 Siehe dazu journalistisch u. a. Kolb 2012.

2 Beispielhaft und ohne jedweden Anspruch auf Vollständigkeit siehe etwa Smith 2006, Hohenstein 2007, Kallen 2009, Smith u. a. 2010, Baldino/Kreider 2011 u. v. a. 


\section{Genese und Regeln des US-amerikanischen Systems der Wahlkampffinan- zierung}

Seit rund vier Jahrzehnten gibt es intensive Bemühungen, das US-amerikanische System der Wahlkampffinanzierung mit vergleichsweise strengen Limitierungen zu versehen. Das zentrale Gesetz des Federal Election Campaign Act (FECA) stammt aus dem Jahr 1972 und führte 1974 zur Einrichtung der Federal Election Commission (FEC), welche die Einhaltung der Finanzierungsgrenzen - damals 1.000 USDollar für Einzelpersonen und 5.000 US-Dollar für PACs, während Unternehmensund Gewerkschaftsgeld als corporate money verboten war - überwachen sowie alle Finanzströme sichtbar machen sollte. Damit hoffte man vermögende Spender in ihren Einflussmöglichkeiten zu limitieren. Das bezog sich freilich nur auf Direktspenden für Kandidaten (hard money), während Firmen und Interessenvertretungen prompt PACs gründeten - Gewerkschaften waren deren „Erfinder“-, und auf dem indirekten Weg der Parteispenden und Kostenübernahme von Wahlkampfaktivitäten (soft money) de facto ohnehin keine Grenzen galten.

Die Spendenlimits nahmen sich im Verhältnis zu den realen Wahlkampfausgaben bald geradezu lächerlich aus, selbst wenn man die Möglichkeit von Vielfachspenden in zahlreichen Wahlkämpfen mit jeweils mehreren Kandidaten berücksichtigt. Die Parteien wurden zu einem Instrument des organisierten Geldtransfers, weil zudem oft Kandidaten erfolgreiches Fundraising betrieben, die mangels ernsthafter Konkurrenz in ihrem Wahlkreis nicht darauf angewiesen waren. Dieses Geld wurde in umkämpfte Schlüsselbezirke umgeleitet. Reformvorschläge scheiterten in den 1980er und 1990er Jahren im Kongress konsequent.

Erst der Bipartisan Campaign Reform Act (BCRA) 2002 verdoppelte die hard money-Obergrenzen und untersagte dafür soft money-Spenden an nationale Parteiorganisationen, was freilich eine Transferrolle der einzelstaatlichen bzw. regionalen und lokalen Parteien forcierte. Zudem wurde vorübergehend die Option gestrichen, dass Unternehmen und Gewerkschaften für elektronische Kommunikation vulgo Werbung im Fernsehen unter dem euphemistischen Deckmantel des electioneering in Wahrheit Wahlkämpfe finanzierten. Anfangs bestätigten die Gerichte größtenteils die Verfassungskonformität des BCRA, wobei es allerdings laufend eine Suche nach Schlupflöchern - so eine bezahlte Dokumentation über Hillary Clinton als Non-Werbung und Auslöser des Letzturteils 2010 - gab.

2010 entschied der U.S. Supreme Court im Fall Citizens United vs. Federal Election Commission (Mason/James 2011; Briffault 2011), dass aufgrund des höherwertigen Rechtsgutes der Meinungsfreiheit unabhängige Ausgaben (independent expenditures) von organisierten Interessen für politische (Fernseh-)Werbung nicht 
untersagt werden dürfen. Es ist nicht einmal erforderlich klar auszuweisen, wer und welche Interessen hinter den Geldgebern stehen, solange keine direkte Absprache der Werbemaßnahmen mit den Kandidaten erfolgt. Solche Außenmittel (outside spending) erhöhten sich von unter 70 Millionen US-Dollar im Jahr 2008 auf fast 170 Millionen allein bis zum 30. Juni 2012, das heißt noch vor Beginn der eigentlichen Intensivphase des Wahlkampfs. Top-Gruppen wie für Mitt Romney Restore Our Future und für Newt Gingrich Winning Our Future mit Ausgaben im jeweils höheren zweistelligen Millionenbereich stellen auf den ersten Blick Worthülsen dar und sind nicht transparent zuzuordnen. Die Mehrheit von insgesamt 640 sogenannter Super-PACs unterstützte übrigens republikanische (Vor-)Wahlkandidaten für die Präsidentschaft.

Schon viel früher (1976) hatte das Höchstgericht in seinem Urteil Buckley vs. Valeo entschieden, dass kein Kandidat daran gehindert werden kann, unbeschränkt persönliche Geldmittel für seine Wahl einzusetzen, solange er nicht - in Präsidentschaftswahlen möglich - eine öffentliche Finanzierung annimmt und dadurch deren Rahmenbedingungen akzeptiert. Die besondere Betonung der freien MeinungsäuBerung als Verfassungsinterpretation ist in den USA geschichtlich zu verstehen immerhin handelte es sich um eine Auswanderergesellschaft, welche neben wirtschaftlichen Gründen aufgrund schlechter Erfahrungen mit einer nicht-demokratischen Obrigkeit entstand -, führt jedoch polemisch ausgedrückt zu einer paradoxen Situation: Es ist verboten, einem Kandidaten 100.000 US-Dollar für den Wahlkampf zu überweisen, doch kann man relativ problemlos Millionen für die mediale Verkündung ausgeben, warum jemand der am besten geeignete Kandidat wäre. Dasselbe gilt für Negativkampagnen - dass der jeweilige Widersacher unqualifiziert wäre -, was oft der eigentliche Kommunikationsinhalt von Super-PACs ist.

\section{a) Limitierung der Wahlkampfspenden}

Derzeit - 2011/12 (siehe Tabelle 1) - gelten in den USA für die Wahlkampffinanzierung nachstehende Spendenlimits:

- Einzelpersonen dürfen pro Wahlzyklus einem Kandidaten nicht mehr als 2.500 US-Dollar und in Summe für alle Kandidaten nicht mehr als 46.200 US-Dollar spenden. Hinzukommen bis zu 30.800 Dollar im Kalenderjahr für nationale und 10.000 Dollar für regionale oder lokale Parteikomitees. Anderen politischen (Aktions-)Komitees können maximal 5.000 US-Dollar gespendet werden. 
Tabelle 1: Spendenlimits in US-Wahlkämpfen 2011/12

\begin{tabular}{|c|c|c|c|c|c|}
\hline & $\begin{array}{l}\text {... to each } \\
\text { candidate or } \\
\text { candidate } \\
\text { committee } \\
\text { per election }\end{array}$ & $\begin{array}{l}\text {... to natio- } \\
\text { nal party } \\
\text { committee } \\
\text { per calen- } \\
\text { dar year }\end{array}$ & $\begin{array}{c}\text {... to state, } \\
\text { district \& lo- } \\
\text { cal party } \\
\text { committee } \\
\text { per calendar } \\
\text { year }\end{array}$ & $\begin{array}{l}\ldots . \text { to any } \\
\text { other politi- } \\
\text { cal commit- } \\
\text { tee per ca- } \\
\text { lendar year }\end{array}$ & Special Limits \\
\hline $\begin{array}{l}\text { Individual may } \\
\text { give }\end{array}$ & $\$ 2,500^{*}$ & $\$ 30,800^{*}$ & $\begin{array}{c}\$ 10,000 \\
\text { (combined li- } \\
\text { mit) }\end{array}$ & $\$ 5,000$ & $\begin{array}{l}\$ 117,000 * \text { overall } \\
\text { biennial limit } \\
\text { (\$46,200* to all } \\
\text { candidates, } \\
\$ 70,800 * \text { to all } \\
\text { PACs \& parties) }\end{array}$ \\
\hline $\begin{array}{l}\text { National Party } \\
\text { Committee may } \\
\text { give }\end{array}$ & $\$ 5,000$ & No limit & No limit & $\$ 5,000$ & $\begin{array}{l}\$ 43,100 * \text { to Sen- } \\
\text { ate Candidates per } \\
\text { campaign }\end{array}$ \\
\hline $\begin{array}{l}\text { State, District \& } \\
\text { Local Party Com- } \\
\text { mittee may give }\end{array}$ & $\begin{array}{c}\$ 5,000 \\
\text { (combined li- } \\
\text { mit) }\end{array}$ & No limit & No limit & $\begin{array}{c}\$ 5,000 \\
\text { (combined li- } \\
\text { mit) }\end{array}$ & No limit \\
\hline $\begin{array}{l}\text { PAC (multicandi- } \\
\text { date) may give }\end{array}$ & $\$ 5,000$ & $\$ 15,000$ & $\begin{array}{c}\$ 5,000 \\
\text { (combined li- } \\
\text { mit) }\end{array}$ & $\$ 5,000$ & No limit \\
\hline $\begin{array}{l}\text { PAC (not multi- } \\
\text { candidate) may } \\
\text { give }\end{array}$ & $\$ 2,500^{*}$ & $\$ 30,800^{*}$ & $\begin{array}{c}\$ 5,000 \\
\text { (combined li- } \\
\text { mit) }\end{array}$ & $\$ 5,000$ & No limit \\
\hline $\begin{array}{l}\text { Authorized Cam- } \\
\text { paign Committee } \\
\text { may give }\end{array}$ & $\$ 2,000^{5}$ & No limit & No limit & $\$ 5,000$ & No limit \\
\hline
\end{tabular}

* Automatischer Inflationsausgleich

Quelle: Center for Responsive Politics (CRP), http://www.opensecrets.org/overview/limits.php (Stand: 30.6.2012).

- Ähnliche Grenzen von 2.500 und 5.000 US-Dollar gelten für alle Arten politischer (Partei-)Komitees, was Direktgelder an Kandidaten betrifft. Geldflüsse innerhalb von Parteikomitees sind ohne Obergrenze möglich.

- PACs unterscheiden sich in ihrer Unterstützung für einzelne oder mehrere Kandidaten. Je nachdem dürfen 2.500 oder 5.000 US-Dollar an Einzelkandidaten und 15.000 oder 30.800 Dollar an nationale Parteikomitees gegeben werden. Für andere Komitees sind 5.000 US-Dollar die Grenze. Anders als für Einzelpersonen fehlt ein Höchstbetrag, so dass theoretisch die höchstmögliche Kandidatenspende in Wahlkämpfen für das Repräsentantenhaus mit 435 Wahlbezirken und deren 
durchschnittlicher Kandidatenzahl multiplizierbar ist, was zu Millionenbeträgen führen kann.

- Für offizielle Komitees eines Kandidaten gelten dieselben Grenzen wie für ihn unterstützende PACs.

Nicht erfasst werden (nach einem Finanzrechtsparagraphen benannte) „527““-Komitees bzw. -Gruppen, die unbeschränkt Geld für politische Aktivitäten einnehmen und ausgeben dürfen, so etwa zur Wählermobilisierung. Lediglich die unmittelbare Unterstützung eines spezifischen Kandidaten ist nicht zulässig, was Grauzonen fördert. Für die aktuellen Kongress- und Präsidentschaftswahlen sind bis zum 30. Juni 2012 unter den führenden 100 Geldgebern prompt praktisch alle Top-Unternehmen und großen Gewerkschaften der USA vertreten. ${ }^{3}$

Eine öffentliche Wahlkampffinanzierung existiert, wie erwähnt, für Kongresswahlen nicht. Seit den Wahlen 1976 gibt es hingegen für Präsidentschaftskandidaten öffentliche Wahlkampfgelder, welche die demokratietheoretischen Ideale der ökonomischen Unabhängigkeit und Chancengleichheit von Kandidaten sichern soll (Filzmaier/Plasser 2002). Wer in den Vor- und/oder Hauptwahlen öffentliche Wahlkampfmittel akzeptiert, ist jedoch bei der Annahme von privaten Spenden an die skizzierten Beschränkungen gebunden. Auch dürfen neben der Inanspruchnahme öffentlicher Mittel maximal 50.000 US-Dollar aus dem Privatvermögen verwendet werden. Ein Verzicht auf solche Mittel ermöglicht hingegen - als durch das erwähnte höchstgerichtliche Urteil Buckley vs. Valeo gesichertes Recht - den unbeschränkten Einsatz des Privatvermögens oder auch von aus Krediten stammenden Geldern. Eine Ausweitung der öffentlichen Wahlkampffinanzierung würde demzufolge, weil in jedem Fall betragsmäßig limitiert, die Korrelation von Reichtum und politischen Erfolgschancen vermögender Quereinsteiger verstärken.

Für den Wahlkampf im engeren Sinn, das heißt nach den parteiinternen Vorwahlen, beziehen die von den Hauptparteien nominierten Präsidentschaftskandidaten Geld durch die FEC, das ihre Wahlkampfausgaben decken soll (block grants). Wird das Geld akzeptiert, darf im Wahlkampf-das heißt ab dem Zeitpunkt der offiziellen Nominierung der Kandidaten auf den Bundesparteitagen, die im Spätsommer stattfinden - keine höhere Summe ausgegeben werden. Kandidaten von Drittparteien können nach der Wahl, wenn sie mehr als fünf Prozent der Stimmen erreicht haben, eine Rückerstattung von Wahlkampfausgaben beantragen. Realiter verstärkt diese Regelung die Chancenlosigkeit von Kandidaten der Drittparteien und von unabhängigen Bewerbern, weil lediglich in Ausnahmefällen - siehe den Milliardär Ross

3 Siehe dazu die Angaben des Center for Responsive Politics (CRP), verfügbar unter http://www.op ensecrets.org/overview/topcontribs.php (Stand: 2.7.2012). 
Perot 1992 und 1996, der den Wahlkampf aus Privatmitteln bestritt - das finanzielle Risiko getragen werden kann.

\section{b) Realpolitische Grauzonen}

Einerseits dürfen wie beschrieben ,527“-Gruppen für unabhängige Nicht-Wahlkampfaktivitäten unbeschränkt Geld ausgeben. Unter Ihnen befindet sich prompt ein illustres Spektrum von Investmentbanken über Spielcasinos bis hin zu Mäzenen. Andererseits werden beispielsweise die Ausgaben im Rahmen der Bundesparteitage nicht erfasst, weil sie aus formaler Sicht keine unmittelbare Wahlwerbung darstellen. Politisch steht freilich auf dem Parteitag keine inhaltliche Entscheidung, sondern bloß die medienwirksame Nominierung des jeweiligen Präsidentschaftskandidaten im Mittelpunkt. Die Kosten eines Parteitages werden auf bis zu 100 Millionen US-Dollar geschätzt. Grenzfälle, etwa Spenden an Parteien für die Vereinfachung des Registrierungs- oder Vorwahlverfahrens mit dem Argument einer Verbesserung der Demokratiequalität, sind zusätzlich vorprogrammiert. Im Zusammenhang mit der fortschreitenden Internationalisierung und Globalisierung - Stichwort: multinationale Konzerne - ist auch das Verbot von ausländischen Geldmitteln kaum zu überprüfen. Es häufen sich Vorwürfe, dass Parteikomitees illegale Parteispenden von ausländischen Quellen, insbesondere von Vertretern asiatischer Geschäftsinteressen, erhalten und für Wahlkämpfe zur Verfügung gestellt haben.

Auch die direkte Platzierung von sogenannten issue advocacy ads durch einem Kandidaten verbundene Gruppen und Verbände zur Wählermobilisierung spielt in den Wahlkämpfen eine bedeutsame Rolle und ist nicht erst seit dem Citizen United-Urteil ein bedeutsamer Faktor. Ausgangspunkt der Entwicklung war unter anderem eine Innovation von Dick Morris, Wahlkampfmanager für Bill Clinton. Morris hatte 1992 angeregt, zwecks Umgehung der gesetzlichen Spendenlimits für Kandidaten eine Finanzierung von personenbezogener Fernsehwerbung durch Parteien unter Berufung auf deren Meinungsfreiheit zu versuchen.

Das Höchstgericht hat das issue advocacy für Parteien zusätzlich abgesichert, weil im Fall Colorado Republican Federal Campaign Committee vs. Federal Election Commission dem Kongress unabhängig vom Ausmaß der Koordination mit den Wahlkampfteams der Kandidaten untersagt wurde, Parteiausgaben für die jeweilige Wahl zu beschränken. Allerdings wurde diese Entscheidung von den Richtern, welche die Mehrheitsmeinung vertraten, unterschiedlich begründet, nämlich interessanter- und paradoxerweise unter anderem sowohl mit einer Zurückweisung der Vermutung, dass Parteiausgaben in allen Fällen mit den Kandidaten koordiniert 
würden, als auch mit der praktischen Unmöglichkeit, die Ausgaben der Parteien im Wahlkampf von den unbeschränkten Ausgaben des Kandidaten zu unterscheiden. ${ }^{4}$

Im Regelfall handelt es sich bei issue advocacy um Medien(Fernseh-)werbung, doch ist genauso eine Verteilung von Informationsmaterialien sogar in Kirchen und anderswo denkbar. Von politik- und kommunikationswissenschaftlichem Interesse ist die Frage, inwiefern Interessengruppen durch issue advocacy Wahlkämpfe als Podium bzw. Plattform für ihre (Mobilisierungs-)Politik benutzen, um als dritter Akteur neben den Politikern und Medien die Themenlandschaft in der öffentlichen Diskussion zu bestimmen.

\section{Amtsinhaberbonus als post-elektorale Scheindemokratie?}

Im Fall von Finanzierungsvorteilen eines Kandidaten (outspending), die das Doppelte der Mittel des Gegenkandidaten überschreiten, kann unabhängig von anderen Faktoren nicht von einem kompetitiven Wahlkampf gesprochen werden. Von 1970 bis Mitte der 2000er-Jahre erhöhten sich jedoch das durchschnittliche Verhältnis der Finanzmittel für Amtsinhaber und Herausforderer von 1,5 zu 1 in Repräsentantenhaus- und Senatswahlen auf nahezu 5 zu 1 und ist seitdem stagnierend oder bestenfalls leicht rückläufig. ${ }^{5}$ In vielen Fällen verfügte der Herausforderer über keine bzw. weniger als 5.000 US-Dollar Finanzmittel (financially unopposed). Amtsinhaber werden also nicht zuletzt aufgrund ihrer Geldvorteile mit sehr hoher Wahrscheinlichkeit wiedergewählt; ein Trend, der ebenfalls durch die Teledemokratisierung und verstärkte Kandidatenorientierung - Bekanntheitsgrad bzw. Medienecho als Amtsinhabervorteil (incumbency bonus) - begünstigt wurde.

Erneut kandidierende Amtsinhaber im Repräsentantenhaus haben eine Wiederwahlquote von über 98 Prozent und im Senat von bis zu rund 90 Prozent. $^{6}$ In der Politikwissenschaft hat sich dafür der Fachbegriff einer post electoral politics (Filzmaier/Plasser 2005 a) eingebürgert. Das führt zwangsläufig zu einer Fokussierung des Wettbewerbs auf wenige Schlüsselwahlkämpfe bzw. nach Rücktritten oder Todesfällen offenen Sitzen. Diese sind auch besonders kostenintensiv. Für die teuersten Senatswahlkämpfe 2012 in Massachusetts und Texas wurden bis Ende Juni schon über 30 Millionen US-Dollar eingeworben, für Schlüsselwahlkämpfe im Re-

4 Die obigen und nachstehenden Ausführungen finden sich auch in Filzmaier/Plasser 2002 und Filzmaier/Plasser 2005 b.

5 Die Basis sind Berechnungen des Autors ausgehend von Filzmaier/Plasser 2005 a.

6 Eigene Berechnungen nach Davidson/Oleszek/Lee 2011 und den vorangegangen Ausgaben desselben Buches. 
präsentantenhaus - etwa im achten Wahlbezirk Ohios - immerhin zum selben Zeitpunkt bis zu knapp 15 Millionen. $^{7}$

Abbildung 2: Wahlkampfspenden für Amtsinhaber und Herausforderer, Kongresswahlen 2012

\begin{tabular}{|l|c|c|c|}
\hline \multicolumn{3}{|c|}{ Senate } \\
\hline Type of Candidate & Total Raised & $\begin{array}{c}\text { Number } \\
\text { of Candidates }\end{array}$ & Average Raised \\
\hline Incumbent & $\$ 208,778,503$ & 25 & $\$ 8,351,140$ \\
\hline Challenger & $\$ 78,538,547$ & 115 & $\$ 682,944$ \\
\hline Open Seat & $\$ 114,303,627$ & 94 & $\$ 1,215,996$ \\
\hline Grand Total & $\$ \mathbf{\$ 4 0 1 , 6 2 0 , 6 7 7}$ & $\mathbf{2 3 4}$ & $\mathbf{\$ 1 , 7 1 6 , 3 2 8}$ \\
\hline
\end{tabular}

\begin{tabular}{|l|c|c|c|}
\hline \multicolumn{4}{|c|}{ House } \\
\hline Type of Candidate & Total Raised & $\begin{array}{c}\text { Number } \\
\text { of Candidates }\end{array}$ & Average Raised \\
\hline Incumbent & $\$ 412,538,436$ & 418 & $\$ 986,934$ \\
\hline Challenger & $\$ 93,750,701$ & 667 & $\$ 140,556$ \\
\hline Open Seat & $\$ 90,046,206$ & 324 & $\$ 277,920$ \\
\hline Grand Total & $\$ \mathbf{5 9 6 , 3 3 5 , 3 4 3}$ & $\mathbf{1 , 4 0 9}$ & $\mathbf{\$ 4 2 3 , 2 3 3}$ \\
\hline
\end{tabular}

Basierend auf Daten der FEC vom 3. Juli 2012.

Quelle: Center For Responsive Politics (CRP), http://www.opensecrets.org (Stand: 30.6.2012).

Bis zum 30. Juni 2012 wurden von Amtsinhabern in Senatswahlkämpfen durchschnittlich über 8,3 Millionen US-Dollar Wahlkampfgelder eingenommen, von Herausforderern lediglich rund 682.000 US-Dollar. Das entspricht einem Missverhältnis von mehr als $12 \mathrm{zu}$ 1, was jedweden Wettbewerbscharakter verhindert (siehe Tabelle 2). In Summe verfügten Amtsinhaber bereits lange vor dem eigentlichen Hauptwahlkampf im Herbst über mehr als 200 Millionen US-Dollar. Über ein Viertel des gesamten Fundraising wurde für offene Sitze betrieben. Im Repräsentantenhaus ist das beschriebene Verhältnis mit grob gerundet $7 \mathrm{zu} 1$ etwas geringer ausgeprägt. Nichtsdestoweniger besaß zum gleichen Zeitpunkt der durchschnittliche Amtsinhaber fast eine Million US-Dollar in der Kriegskasse, ein Herausforderer im Mittelwert jedoch nur 140.000 US-Dollar. Senatswahlkämpfe sind also generell viel

7 Siehe http://www.opensecrets.org/overview/topraces.php (Stand: 4.7.2012). 
teurer; zu berücksichtigen ist allerdings auch, dass es im Repräsentantenhaus oft keinen Gegenkandidaten gibt und sich dies mangels Wahlkampf bei der Berechnung von Durchschnittswerten auswirkt. Etwas unter einem Viertel des Fundraising in Summe und somit prozentuell ähnlich dem Senat ist allerdings der Aufwand für offene Sitze.

\section{Exkurs: Wahlverhalten als sozio-ökonomische Kandidatenauswahl}

Parallel zu Wettbewerbsvorteilen für finanzstarke Kandidaten zeigt sich, dass das Wahlverhalten und das jährliche Durchschnittseinkommen in engem Zusammenhang stehen (siehe Tabelle 3). Je höher die Einkommensklasse, desto mehr Stimmen erhalten Kandidaten der Republikaner bzw. desto weniger Stimmen gibt es für Kandidaten der Demokraten. Das Spektrum der Abweichung reichte 2010 von 57 Prozent der Stimmen für demokratische Kandidaten von Wählern mit einem Familieneinkommen von weniger als 30.000 US-Dollar im Jahr und nur 43 Prozent bei einem Einkommen von über 100.000 US-Dollar. Die Vergleichswerte für republikanische Kandidaten sind diametral und betrugen 40 bzw. 56 Prozent. Diese Unterschiede werden noch deutlicher, wenn man besonders gut Verdienende mit über 200.000 US-Dollar heranzieht, doch bereits in der Mittelschicht ab einem Einkommen von 50.000 US-Dollar im Jahr entscheiden sich mehr Wähler für republikanische als für demokratische Kandidaten.

Korreliert man das Familieneinkommen mit der ethnischen Herkunft, so werden Republikaner generell mehr von Weißen, jedoch - 2010 im Verhältnis von $61 \mathrm{zu}$ 37 Prozent - vor allem von der wohlhabenden weißen Bevölkerung gewählt. Die Demokraten punkten umgekehrt bei schlechter verdienenden Afro-Amerikanern und Hispanics, welche allerdings einen kleineren Wähleranteil stellen und oft eine niedrigere Wahlbeteiligung aufweisen. 
Tabelle 3: Wahlverhalten nach Einkommensgruppen in den US-Kongresswahlen 2010 (Repräsentantenhaus)

\begin{tabular}{|l|c|c|c|c|}
\hline Family Income (per Year) & Democrat & Republican & Others/No Answer & Share of Vote \\
\hline under US-\$30.000 & 57 & 40 & 3 & 17 \\
$30.000-50.000$ & 51 & 46 & 3 & 19 \\
$50.000-75.000$ & 45 & 51 & 4 & 21 \\
$75.000-100.000$ & 42 & 56 & 2 & 15 \\
$100.000-200.000$ & 43 & 56 & 1 & 8 \\
Over 200.000 & 34 & 64 & 2 & 36 \\
\hline Less Than \$50K & 54 & 43 & 3 & 64 \\
\$50K or More & 42 & 55 & 3 & 73 \\
\hline Less Than \$100K & 49 & 48 & 3 & 27 \\
\$100K or More & 40 & 58 & 2 & 36 \\
\hline Less Than \$50,000 & 54 & 43 & 3 & 27 \\
\$50-100,000 & 44 & 53 & 3 & 25 \\
\$100,000 or More & 40 & 58 & 2 & 11 \\
\hline Whites Under \$50,000 & 42 & 54 & 4 & 11 \\
Whites Over \$50,000 & 37 & 61 & 2 & 1 \\
Non-Whites Under \$50,000 & 80 & 19 & 2 & 37 \\
Non-Whites Over \$50,000 & 69 & 29 & & 2 \\
\hline
\end{tabular}

Quelle: Voter News Service (VNS), Exit Polls, verfügbar unter http://edition.cnn.com/ELECTION/ 2010/results/polls (Stand: 30.6.2012).

\section{Schlussbemerkung}

Das Grunddilemma in den USA ist, dass das System der Wahlkampffinanzierung ein qualitatives Element der Demokratie(-qualität) darstellt, während die gegenwärtigen und zukünftigen Gesetze sich zwangsläufig allein an quantitativen Messungen orientieren müssen, so dass gesetzliche Neuregelungen nicht zu einer vollständigen Problemlösung führen können. Zwei weitere und in EU-ropa naheliegende Ansatzpunkte zu einer grundlegenden Reform bleiben in der US-amerikanischen Diskussion weitgehend ausgeblendet: Die Einführung einer öffentlich-privaten Mischfinanzierung von Kongresswahlkämpfen und öffentlich subventionierte Gratis-Sendezeiten in Rundfunk und Fernsehen in Verbindung mit strikten Begrenzungen der Werbeausgaben. Weil das der US-Philosophie des politischen Denkens und des freien Wettbewerbs widerspricht, ist bei der prinzipiellen Problematik einer komplexen Finanzierung von Wahlkämpfen mit zahlreichen gesetzlichen Schlupflöchern und „Taschen voller Tricks“(Rosenberg 1996) keine Änderung zu erwarten. 


\section{Literatur}

Baldino, Thomas J./Kreider, Kyle L., 2011: U.S. Election Campaigns. A Documentary and Reference Guide, Santa Barbara.

Briffault, Richard, 2011: Symposium Citizens United versus Federal Election Commission. Implications for the American Political Process - Corporations, Corruptions, and Complexity: Campaign Finance after Citizens United, in: Cornell Journal of Law and Public Policy 643 (20) (Special Issue).

Davidson, Roger H./Oleszek, Walter J./Lee, Frances E., 2011: Congress and Its Members, Washington D.C. (13 ${ }^{\text {th }}$ Ed.).

Filzmaier, Peter/Plasser, Fritz, 2002: Campaign Finance in den USA, in: Österreichische Zeitschrift für Politikwissenschaft (1), 41-60.

Filzmaier, Peter/Plasser, Fritz, 2005 a: Post Electoral-Politics und Plutokratie? Die US-amerikanischen Kongresswahlen 2004, in: Zeitschrift für Parlamentsfragen (2), 243-259.

Filzmaier, Peter/Plasser, Fritz, 2005 b: Politik auf amerikanisch. Wahlen und politischer Wettbewerb in den USA, Wien.

Hohenstein, Kurt, 2007: Coining Corruption. The Making of the American Campaign Finance System, Chicago.

Kallen, Thomas P. (Hrsg.), 2009: Campaign Finance: Background, Regulation and Reform, New York.

Kolb, Matthias, 2012: Wenn Politiker nur ans Geld denken, 30.5.2012, http:// www.sueddeutsche.de/politik/wahlkampf-finanzierung-in-den-usa-wenn-politiker-nur-an-geld-denken-1.1368745 (Stand: 30.6.2012).

Mason, Thomas O./James, Sophie A., 2011: Campaign Finance and the Citizens United Supreme Court Case, New York.

Rosenberg, Liza, 1996: A Bag of Tricks. Loopholes in the Campaign Finance System. Washington D.C.

Smith, Melissa M./Williams, Glenda C./Powell, Larry/Copeland, Gary A., 2010: Campaign Finance Reform. The Political Shell Game, Lanham.

Smith, Rodney A., 2006: Money, Power, \& Elections: How Campaign Finance Reform Subverts American Democracy, Baton Rouge.

West, Darrell M., 2000: Checkbook Democracy. How Money Corrupts Political Campaigns, Providence. 
Korrespondenzanschrift:

Univ.-Prof. Dr. Peter Filzmaier

Plattform Politische Kommunikation

Donau-Universität Krems

Dr. Karl Dorrek-Straße 35

A-3500 Krems

E-Mail: peter.filzmaier@donau-uni.ac.at

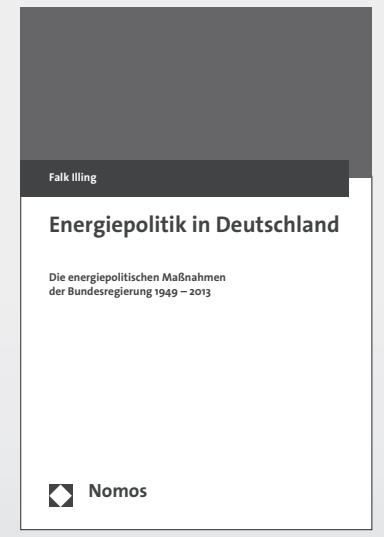

Energiepolitik in Deutschland

Die energiepolitischen Maßnahmen

der Bundesregierung 1949 - 2013

Von Falk Illing

2012, 281 S., brosch., 49,- $€$

ISBN 978-3-8329-7585-2

Die Energiewende ist das umstrittenste Projekt der Bundesregierung. Doch nicht nur die aktuelle Energiepolitik birgt Zündstoff in sich, sondern sie führte seit den Anfängen der Bundesrepublik zu gesellschaftlichen Kontroversen. Diese Studie bietet erstmals einen vollständigen Überblick der Ziele, Maßnahmen und Schwerpunkte der Energiepolitik seit 1949. 